\title{
Study on purification effect of infiltration materials to pavement runoff pollution
}

\author{
Xueling $\mathrm{Xu}^{1}$, Aihong Kang ${ }^{1, \mathrm{a}}$, Zhingping $\mathrm{Lu}^{1}$,Keke lou ${ }^{1}$, Changjiang Kou ${ }^{1}$ \\ ${ }^{1}$ College of Civil Science and Engineering, Yangzhou University, Yangzhou 225127, China
}

\begin{abstract}
The concentrations of pollutants in pavement runoff are higher than those in other surface runoff normally, which causes serious problems in protecting the environment of receiving rivers and soils. According to the concept of low impact development (LID), a pavement runoff pollution control measure was developed by using infiltration structure. In order to infiltration structure play a superior role in the control of pavement runoff pollution, purification capacity of four typical infiltration materials was tested. Then, long-term purification efficiency of infiltration materials was explored to suspended solids. Besides, microscopic observation was performed on infiltration materials. Results indicated that purification effects of infiltration materials were significantly different. The purification process can be divided into three stages, which ware "rapid upgrading ", "slow upgrading " and "slow reduction ". Finally, Infiltration materials mainly have two modes to intercept runoff pollutants, which were pore adsorption and mechanical interception of the gap between materials.
\end{abstract}

\section{Introduction}

With deepening of urbanization process, the proportion of impervious surface increases rapidly, leads sharp reduction of rainwater infiltration rate and great increasing of total amount of runoff. Early research had shown that the concentration of pollutants in pavement runoff had far exceeded the limitation required in the standards, especially in the initial runoff [1-3]. The pollutants were presented by sampling and analyzing the pavement runoff at different spots along the Runyang Road South, Yangzhou, China. The EMC of the four sites are showed in the Table 1. The concentration of each pollutant far exceeded the limitation in the Standard of Environmental Quality for Surface Water. It will cause destruction of aquatic ecosystem if discharge into rivers or groundwater directly without purification treatment.

Table 1 . The results of water quality analysis

\begin{tabular}{ccccc}
\hline Sites & $\begin{array}{c}\mathrm{SS} \\
(\mathrm{mg} / \mathrm{L})\end{array}$ & $\begin{array}{c}\mathrm{COD} \\
(\mathrm{mg} / \mathrm{L})\end{array}$ & $\begin{array}{c}\mathrm{TN} \\
(\mathrm{mg} / \mathrm{L})\end{array}$ & $\begin{array}{c}\mathrm{TP} \\
(\mathrm{mg} / \mathrm{L})\end{array}$ \\
\hline Site1 & 434 & 626 & 8.5 & 1.3 \\
Site2 & 212 & 651 & 2.9 & 0.9 \\
Site3 & 621 & 594 & 6.2 & 2.4 \\
Site4 & 297 & 668 & 3.9 & 1.3 \\
\hline Limitation & $\leq 30$ & $\leq 40$ & $\leq 2$ & $\leq 0.2$ \\
\hline
\end{tabular}

Previous studies identify that it's an efficient and feasible measure to purify the urban surface runoff by infiltration structure $[4,5]$. Infiltration structure can improve the infiltration rate and purify runoff pollutants. Besides it is efficient and low-cost. It has received extensive attention of scholars all over the world in recent years. The core of percolation structure is infiltration materials. Environmental mineral materials, such as diatomite, volcanic and so on are widely used with many advantages to purify water, but purification effect and mechanism of infiltration materials are different, which leads to the actual purification effect of infiltration structure unevenly [6-8]. It is essential to study the purification effect and mechanism of infiltration materials.

In this page, simulated pavement runoff was prepared in the laboratory and used in the infiltration test .The removal rates of SS, COD, TP and TN were used to evaluate contamination degree of typical infiltration materials. On that basis, the microscopic method was adopted to study the purification mechanism. Finally, Long-term purification efficiency of infiltration materials to suspended solids was explored. The aim is to reduce non-point pollution and can also contribute to eco-friendly management of rainfall as well as improvement of water quality.

\section{Test materials and test methods}

\subsection{Test materials}

\subsubsection{Infiltration materials}

* Corresponding author: ${ }^{\text {a } 571857346 @ q q . c o m}$ 
Infiltration materials used for infiltration structure should have stable performance, good adsorption, intercept performance, and not release harmful substance. Referencing filter material commonly used in water treatment, the following four kinds of environment mineral materials as the infiltration materials were selected. That is diatomite, volcanic, slag, zeolite, which are shown in Figure 1.

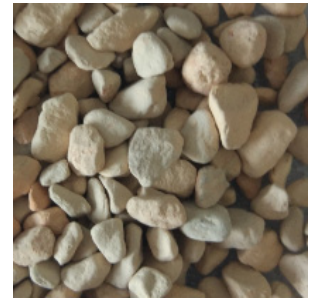

(a) Diatomite

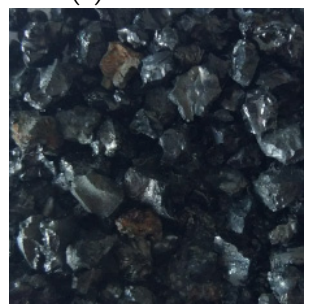

(c) Slag

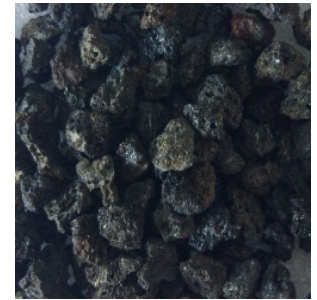

(b) Volcanic

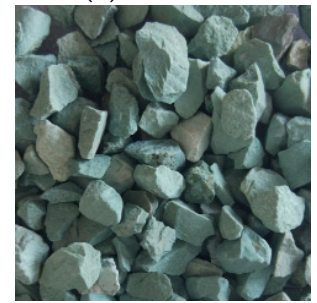

(d) Zeolite
Figure 1. Infiltration materials

\subsubsection{Test water sample}

There is a big difference between water quality test results in different test locations and different levels of rainfall [9]. Some even are differences of orders of magnitude. Therefore, the target concentrations of pollutants were determined according to the existing research results. The method of preparing test runoff is adding reagent in the water [10]. Runoff sample by each pollutant target concentration and the amount of reagent addition are shown in Table 2.

Table 2. Target concentration of pollutant and reagent addition

\begin{tabular}{cccc}
\hline $\begin{array}{c}\text { Item } \\
\mathrm{s}\end{array}$ & Reagent & $\begin{array}{c}\text { Target concentration } \\
(\mathrm{mg} / \mathrm{L})\end{array}$ & $\begin{array}{c}\text { Addition } \\
(\mathrm{g} / 100 \mathrm{~L})\end{array}$ \\
\hline $\mathrm{SS}$ & Soil & 400 & 57.16 \\
$\mathrm{COD}$ & $\mathrm{C}_{6} \mathrm{H}_{12} \mathrm{O}_{6}$ & 650 & 69.80 \\
$\mathrm{TP}$ & $\mathrm{KH}_{2} \mathrm{PO}_{4}$ & 1.5 & 0.55 \\
$\mathrm{TN}$ & $\mathrm{NH}_{4} \mathrm{Cl}$ & 6.0 & 3.62 \\
\hline
\end{tabular}

\subsection{Test methods}

\subsubsection{Device and steps for infiltration test}

In order to measure the purification effect of different infiltration materials, the experiment was carried out in the laboratory by simulation device made by ourselves. The test device is shown in Figure 2. It can not only carry out the purification test of runoff pollution, but also has the function of measuring the permeability coefficient of the materials. There are four overflows on device and the interval is $10 \mathrm{~cm}$, which can control the height of the water according to the thickness of the materials.

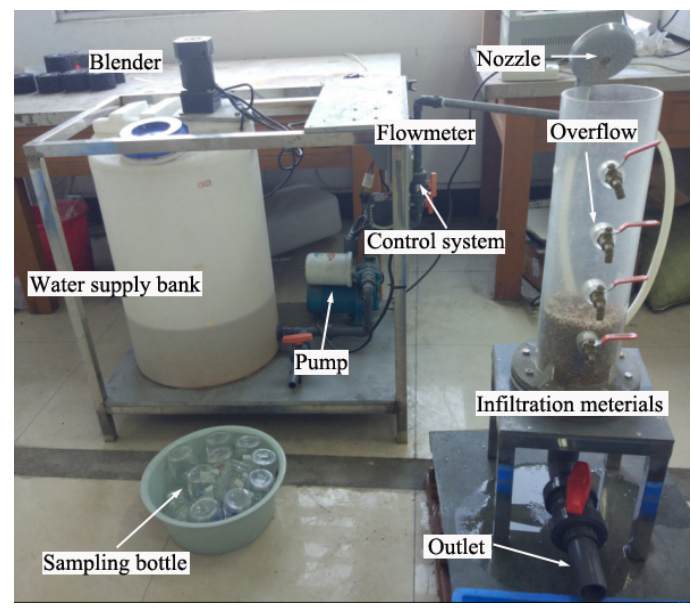

Figure 2. The device of infiltration test

The test steps for the removal rate of pollutants are as follows:

Before the test, it is necessary to wash the materials several times, and then need to be dried.

(1) Place the infiltration materials in the device equably.

(2) Inject the water into the bucket, add the reagent to water and stir fully for preparing the simulation runoff.

(3) Open the overflow and the outlet of the device. The sewage in the bucket is communicated with the device at the same time. Make the sewage flow uniformly in the overflow by adjusting the flowmeter. Collect water sample from the outlet after $2 \mathrm{~min}$.

(4) Determine the concentration of COD, SS, TP and $\mathrm{TN}$ in collecting water samples, the detection methods of pollutant concentration are shown in Table 3.

(5) The pollutants removal rates of four infiltration materials calculated by formula (1).

$$
\eta=\frac{C_{0}-C_{1}}{C_{0}} \times 100 \%
$$

Table 3. Detection methods of pollutant concentration

\begin{tabular}{cc}
\hline Items & Methods \\
\hline $\mathrm{SS}$ & Gravimetric method \\
$\mathrm{COD}$ & Potassium dichromate \\
$\mathrm{TN}$ & UV spectrophotometry \\
$\mathrm{TP}$ & Ammonium molybdate spectrophotometry \\
\hline
\end{tabular}

\subsubsection{Microcosmic test method}

The characteristics and pore structure of different 
infiltration materials are different, and the removal ability and adsorption mechanism to pollutants are also different. In order to analyze the purification mechanism of infiltration materials from the microscopic perspective, the micro morphology was observed before and after the infiltration test by using the XL-30 Environment Scanning Electron Microscope (XL-30ESEM).

\section{Results and discussion}

\subsection{Purification effect of infiltration materials to pavement runoff pollution}

The pollutants removal rates of four infiltration materials are shown in Figure 3. From the category of pollutants, SS is the most easy to remove. The removal rate of SS is maintained at the same level, and the average removal rate is above $70 \%$. The purification effect of four kinds of infiltration materials on COD and $\mathrm{TN}$ are poor, COD The average removal rate of TN was $40 \sim 50 \%$, and the average removal rate of $\mathrm{TN}$ was $25 \sim 35 \%$. The purification effect of infiltration materials on $\mathrm{TP}$ is better, the average removal rate is $35 \sim 60 \%$.

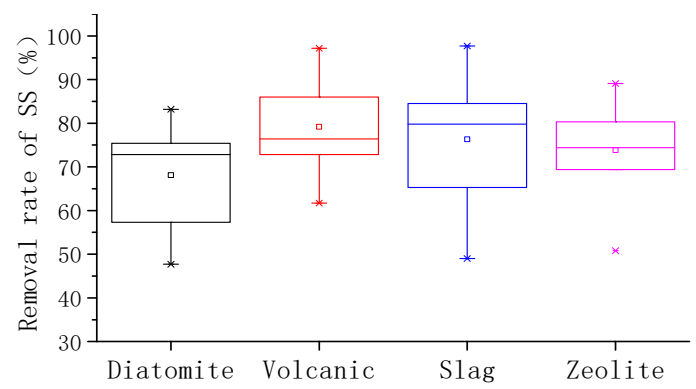

(a) Removal rate of SS

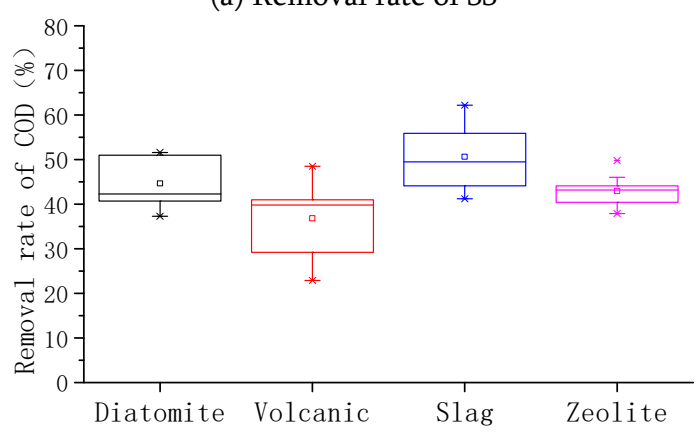

(b) Removal rate of COD

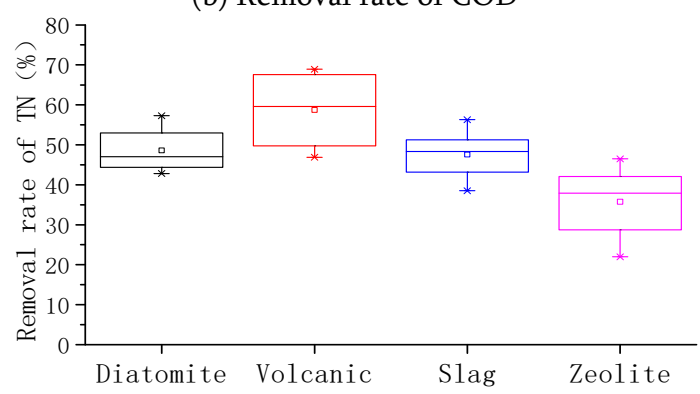

(c) Removal rate of $\mathrm{TN}$

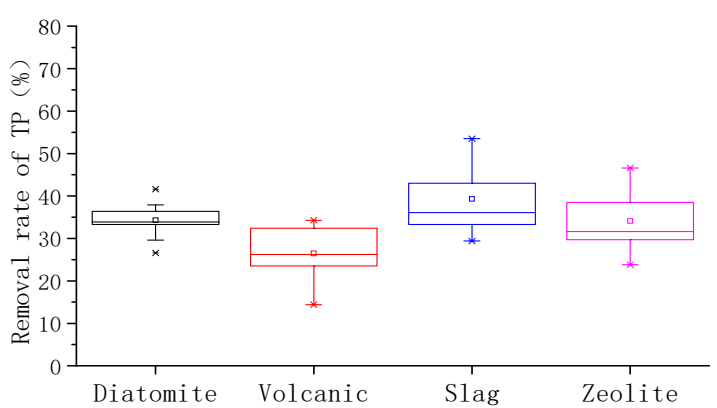

(d) Removal rate of TP

Figure 3. Pollutants removal rates of infiltration materials

From the infiltration materials, the removal efficiency of various materials on pavement runoff pollutants is different. Diatomite has a good removal effect on TP, but it has poor purification effect on Zn, obviously lower than other materials. The removal effect of volcanic on SS and TP is better, especially for TP. The slag has better purification effect on COD and $\mathrm{TN}$. The purification effect of zeolite on TP is poor, and the removal rate of other pollutants is similar to that of other materials. The main reason for this distinction is that the composition, pore structure and pore distribution of materials are different, then lead to the different adsorption capacity and mechanism of different pollutants.

\subsection{Long-term purification efficiency of infiltration materials}

The dissolved pollutants showed the similar spatial distribution with SS because of their high attachment to particles. When the dry weather effect is not considered, the amount of pollutant can be equivalent to the total amount of suspended solids in the corresponding runoff. With the total amount of pollutants as the control index, the average annual rainfall is about $1026 \mathrm{~mm}$, and the runoff of each simulated period can be roughly determined to be about $1026 \times 3.14 \times 50^{2} \times 10^{-6}=8.05 \mathrm{~L}$ 。 After each simulation period (one year), runoff samples were collected to test the pollutant content. The results are shown in Figure 4. 


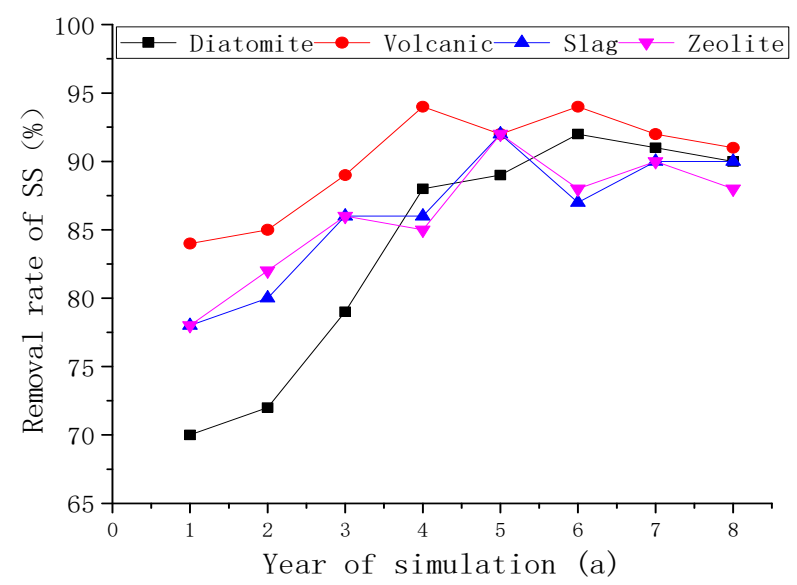

Figure 4. Results of long-term infiltration test

With the increase of the simulation period, the purification rate of pollutants has a certain increase on the whole. In the early stage of simulation, the growth of the pollutants is faster, the growth of the pollutants is slow in the middle and late stage, and some fluctuations appear, and even the purification rate of the pollutants has a slow downward trend in the later period.

\subsection{Purification mechanism of infiltration materials to pavement runoff pollution}

XL-30ESEM is used to observe the microstructure of infiltration materials before and after the purification test, as shown in Figure 4.

The surface of the diatomite and zeolite presents tetrahedral network structure, which forms a good channel between materials. The surface of volcanic is uneven, with rich pore. It is clear that the inner pores are filled with pollutants after the infiltration test, so as to achieve the purification effect. It can be observed from the image that the slag itself contains more porous vitreous, porous carbon and coke. These porous components have good adsorption effect on pollutants. It is obviously observed that Pollutants are adsorbed in the micropore.
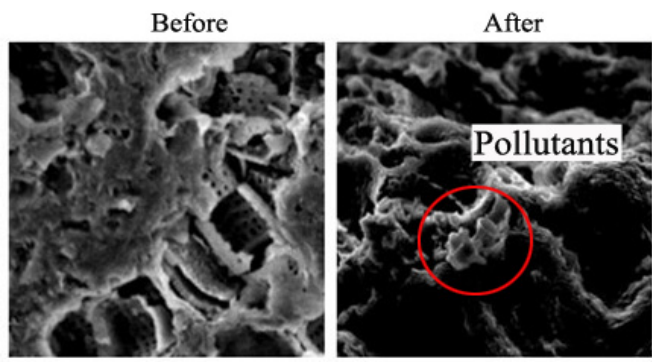

(a) Diatomite

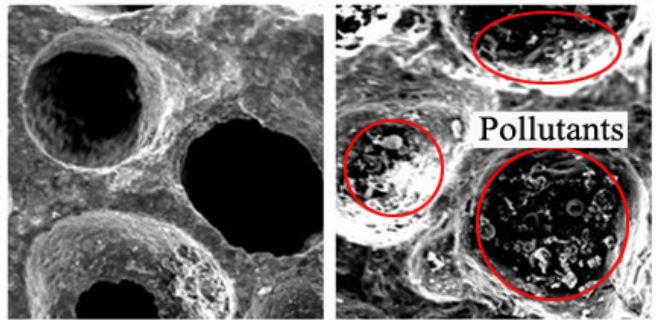

(b) Volcanic

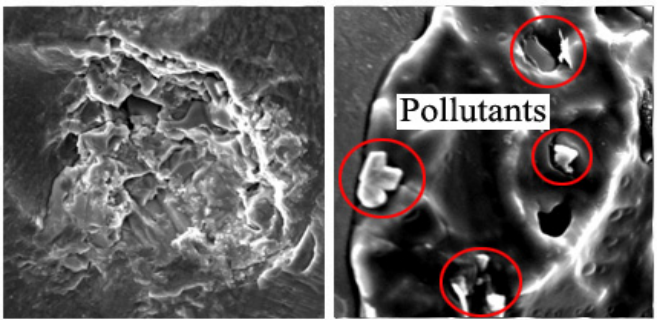

(c) Slag

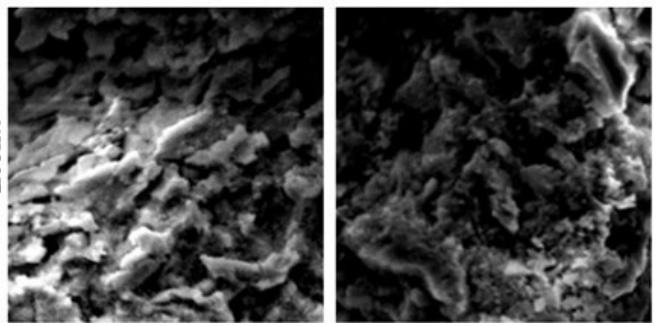

(d) Zeolite

Figure 5. The microstructure of percolation media before and after purification experiment

Therefore, for the purification of pavement runoff pollutants, diatomite and zeolite mainly rely on the mechanical interception of the gap between materials. While volcanic and slag depend on pore adsorption and mechanical interception of the gap between materials. The adsorption characteristic of the porous materials plays a great role in purifying the pollutants in the runoff samples.

\section{Conclusions}

Purification effect of four typical infiltration materials was tested and long-term purification efficiency was explored to suspended solids. Besides, microscopic observation was performed on infiltration materials. The following conclusions can be drawn: Infiltration materials have different purification effects on different pavement runoff pollutants. The long-term purification process can be divided into three stages, which ware "rapid upgrading ", "slow upgrading " and "slow reduction ". Infiltration materials mainly have two modes to intercept runoff pollutants, which were pore adsorption and space interception of structure.

The research result has been partially put into engineering practice, the success of which is of technical significance for the exploration of new pollution control technologies. 


\section{Acknowledgement}

The authors would like to acknowledge the financial support from the National Natural Science Foundation Committee of China (51578481), and the Research and Innovation Plans for Graduates in Jiangsu Province (KYCX17_1880).

\section{References}

1. Masoud K, Boaz D F, John S G, Review of highway runoff characteristics:Comparative analysis and universal implications, Water Research, 46, 66096624 (2012)

2. Mayer T, Rochfort Q, Marsalek J. Environmental characterization of surface runoff from three highway sites in Southern Ontario, Water Quality Research, 46, 110-120 (2011)

3. Modugno M D, Gioia A, Gorgoglione A. BuildUp/Wash-Off Monitoring and Assessment for Sustainable Management of First Flush in an Urban Area, Sustainability, 7, 5050-5070 (2015)

4. Jiake Li, Guangfei Du, Purification effects of biological filter on urban road runoff, Soil and Water Conservation , 4, 1-6 (2012)

5. Davis A P, Shokouhian M, Water quality improvement through bioretention media: Nitrogen and Phosphorus Removal, Water Environment Federation, 78, 284-93 (2006)

6. Laurence W G, Pamela R, Neil M P, Accumulation of heavy metals in a constructed wetland treating road runoff , Ecological Engineering, 70, 133-139 (2014)

7. Huixia Chen, Xiang Liu, Study on the control technology of urban runoff pollution by zeolite infiltration, Environmental Engineering, 6, 519-512 (2012)

8. Xiao L, Suiliang H, Characteristics of phosphorous adsorption on three substrates used in constructed wetland, Environmental Engineering, 6, 3367-3372 (2012)

9. Qianqian Z, Xiangquan L, Source apportionment of pollutants in urban roadway runoff, Ecology and Environmental Sciences, 23, 352-358 (2014)

10. Aihong K, Keke L, Peng X, Yangzhou road surface runoff pollution characteristics analysis and emission law, Highway, 8, 212-216 (2016) 